\title{
Adult Primary Bone Sarcoma and Time to Treatment Initiation: An Analysis of the National Cancer Database
}

\author{
Joshua M. Lawrenz ${ }^{1}$, ${ }^{1}$ Gannon L. Curtis, ${ }^{1}$ Joseph F. Styron, ${ }^{1}$ Jaiben George, ${ }^{1}$ \\ Peter M. Anderson, ${ }^{2}$ Stacey Zahler, ${ }^{2}$ Dale R. Shepard, ${ }^{2}$ Brian P. Rubin, ${ }^{3}$ \\ Lukas M. Nystrom $\mathbb{D}^{1}{ }^{1}$ and Nathan W. Mesko ${ }^{1}$ \\ ${ }^{1}$ Orthopaedic and Rheumatologic Institute, Cleveland Clinic, Cleveland, OH 44195, USA \\ ${ }^{2}$ Taussig Cancer Institute, Cleveland Clinic, Cleveland, OH 44195, USA \\ ${ }^{3}$ Robert J. Tomsich Pathology and Laboratory Medicine Institute, Cleveland Clinic, Cleveland, OH 44195, USA
}

Correspondence should be addressed to Joshua M. Lawrenz; josh.lawrenz@gmail.com

Received 7 August 2018; Accepted 14 October 2018; Published 11 November 2018

Academic Editor: Eugenie S. Kleinerman

Copyright (c) 2018 Joshua M. Lawrenz et al. This is an open access article distributed under the Creative Commons Attribution License, which permits unrestricted use, distribution, and reproduction in any medium, provided the original work is properly cited.

Objective. The time to treatment interval (TTI), defined as the period from diagnosis to first definitive treatment, has very limited descriptions toward understanding delays in primary bone sarcoma (PBS) care. Our primary goal was to determine the national standard for time to treatment initiation (TTI) in PBS in adults and to identify characteristics associated with TTI variability. Methods. An analysis of the National Cancer Database identified 15,083 adult patients with PBS diagnosed from 2004 to 2013. Kruskal-Wallis analysis identified differences between covariates regarding TTI and regression modeling identified covariates that independently influenced TTI. Results. The median TTI was 22 days. Approximately $60 \%$ of patients were definitively treated in the same center where the index diagnosis was made. Increased TTI was correlated with a transition in care institution (incidence rate ratio $($ IRR $)=1.89 ; P<0.001)$, being uninsured $(\mathrm{IRR}=1.36 ; P<0.001)$, primary tumor site in the pelvis $(\mathrm{IRR}=1.26 ; P<0.001)$, Medicaid insurance status $(\mathrm{IRR}=1.22 ; P<0.001)$, care at an academic center $(\mathrm{IRR}=1.14 ; P<0.001)$, non-white race $($ IRR $=1.12$; $P=0.002)$, and Medicare insurance status (IRR $=1.08 ; P=0.017$ ). Decreased TTI was correlated with a diagnosis of chondrosarcoma (IRR $=0.85 ; P<0.001$ ), having surgery as the index treatment (IRR $=0.88 ; P<0.001$ ), a primary tumor site of the lower $(I R R=0.91 ; P=0.001)$ or upper extremity (IRR $=0.92 ; P=0.023)$, and stage II or stage III disease $($ IRR $=0.91 ; P=0.010)$. Conclusions. TTI is associated with tumor, treatment, and socioeconomic and healthcare system characteristics. Transitions in care between institutions are responsible for the greatest increase in TTI. As TTI is more commonly used as a quality metric, physicians need to be aware of the causes for prolonged TTI, as we work to improve national delays in diagnosis and treatment initiation.

\section{Introduction}

On an annual basis, there are approximately 3,500 new patients diagnosed with primary bone sarcoma (PBS) in the United States [1]. While treatment almost always includes surgery, the addition of chemotherapy in the 1980s dramatically increased the survival [2-4]. Similar to other cancer types, it is recommended that treatment be initiated as early in the disease course as possible to reduce the risk of metastatic spread or growth [5-7].
Time to treatment initiation (TTI), defined as the time in days from histologic diagnosis of malignancy to initiation of definitive treatment, is being used as a quality control metric in an effort to improve patient outcomes in cancer referral centers. Prolonged TTI is reported to have a negative survival impact in several cancer types, including breast and head/neck cancer [8-10], lending evidence for expedited treatment strategies. To our knowledge, there have been no similar studies assessing the prognostic influence of TTI in PBS, yet it is a logically accepted mindset to expedite 
diagnosis and treatment with hopes of theoretically improving outcome. Thus, with a limited understanding surrounding the importance of time in the treatment of PBS, it is helpful to identify the risk factors associated with TTI in PBS.

Our primary aim was to quantify the current norms for TTI for PBS in the United States. Additionally, we aimed to identify patient, tumor, treatment, and healthcare-associated characteristics associated with prolonged TTI following a PBS diagnosis.

\section{Methods}

2.1. Database. Following the approval by our IRB, the National Cancer Database (NCDB) was reviewed from 2004 to 2013. Created in 1989 by the American College of Surgeons (ACS) and the Commission on Cancer (CoC), the NCDB captures $70 \%$ of all new United States cancer diagnoses, compiling data from over 1,500 cancer centers [11]. The requirements and methodology for reporting to the NCDB have been described [11-14].

2.2. Selection of Patients. Adult patients ( $\geq 18$ years old) with PBS diagnosed between 2004 and 2013 were identified using topographical codes (C40.0-C40.3, C40.8-C41.4, C41.8, C41.9) designated by International Classification of Disease for Oncology, Third Edition (ICD-O-3). To be included, a patient also required an ICD-O-3 histology code consistent with a PBS. These codes identified a total of 15,083 patients with a PBS. Patients were excluded if they did not receive definitive treatment in the form of surgery, radiotherapy, systemic therapy, or other forms of treatment $(n=1,720)$. Additional patients were excluded if TTI was $>365$ days $(n=34)$ due to potential recording error or patient decision to voluntarily delay treatment.

Patient, tumor, treatment, and healthcare system factors were assessed to identify their relationship to TTI. Patient factors included demographics such as age, gender, race, Charlson/Deyo Score (0, 1 , or $\geq 2)$, and annual income. Tumor and treatment factors included histologic subtype, primary site, size, grade, clinical stage, and initial definitive treatment modality. Healthcare system factors included treating facility type, insurance provider, distance from the patient's residence to the treating facility, and a transition in care institution. Facility types are divided into community cancer programs, comprehensive cancer centers, academic centers, integrated network cancer programs, and others types (i.e., Veteran's Affairs cancer programs). While both offering diagnostic and treatment services, community cancer programs diagnose 100-500 new cancer cases a year, and comprehensive and academic cancer programs diagnose $>500$ new cancer cases a year. "New cancer cases" are defined as all histologic diagnoses-not exclusively sarcoma. Initial definitive treatments include surgery, radiotherapy, systemic therapy, or a combination of treatments that start on the same day. Noncurative treatments were not considered initial definitive treatments-such treatments may include a palliative or hospice approach. Patients who received a diagnosis at one facility and were then transferred to another facility for definitive treatment initiation were considered to have a transition in care institution.

2.3. Statistical Analysis. In order to determine significant variances in TTI within a variable, the Kruskal-Wallis test was used for nonparametric univariate analysis. Multivariate analysis was performed using negative binomial regression models, containing all covariates to control for confounders. $P$ values were considered significant if $P \leq 0.05$. Incidence rate ratio (IRR) is defined in the following manner-for every 1-point increase in the independent variable, while holding all other variables in the model constant, the TTI rate (in days) would increase by a factor of that value. Statistical tests were conducted using Stata Version 14 (College Station, TX).

\section{Results}

In total, 13,329 patients were included in the final analysis (Figure 1). Patients between 51 and 70 years of age (32\%) were most commonly affected by PBS, followed by patients between 31 and 50 years (29\%) and 18-30 years (24\%). Over half of the population was male $(56 \%)$, and the vast majority was either white $(85 \%)$ or black $(9 \%)$. The most common histologic types included chondrosarcoma (42\%), osteosarcoma (30\%), Ewing's sarcoma (10\%), and chordoma (10\%). The lower extremity (34\%), pelvis (19\%), and upper extremity $(13 \%)$ were the most frequent sites of primary tumors. Academic centers (42\%) and comprehensive cancer centers (15\%) reported over half of the cases, while $40 \%$ of cases experienced a transition in care. Surgery (67\%) and systemic treatments (26\%) were the most frequent initial treatments.

In the United States, the median TTI for all patients diagnosed with PBS was 22 days (interquartile range (IQR) 4-43 days), while the mean TTI was 32 days. From 2004 to 2013, median TTI remained relatively constant, with a slight increase in $2013(P=0.165)$ (Figure 2$)$. Univariate analysis revealed significant differences in TTI with regard to patient, tumor, and healthcare factors-including age, gender, race, histologic subtype, primary site, tumor size, grade, clinical stage, facility type, insurance, distance from facility, initial treatment, and transition in care (Table 1). A detailed report of the relative TTI IRR of each of these factors is displayed in Table 2. Figure 3 compares the covariates associated with significantly increased TTI to those covariates associated with significantly shortened TTI.

The single patient factor that was significantly associated with TTI was race. Patients who were a minority race were found to have increased TTI compared to white patients (IRR = 1.12; 95\% confidence interval (CI), 1.04-1.19; $P=0.002)$. Age $(\mathrm{IRR}=1.07 ; 95 \% \mathrm{CI}, 0.94-1.14 ; P=0.051)$, female gender $(\mathrm{IRR}$ $=0.99 ; 95 \%$ CI, 0.94-1.04; $P=0.612$ ), and a Charlson/Deyo Comorbidity Score $\geq 1$ (IRR $=0.98 ; 95 \%$ CI, 0.92-1.06; $P=0.677)$ were not significant predictors of TTI.

Pelvic tumor location $(\mathrm{IRR}=1.26$; 95\% CI, 1.19-1.35; $P<0.001)$ was associated with increased TTI compared to all 


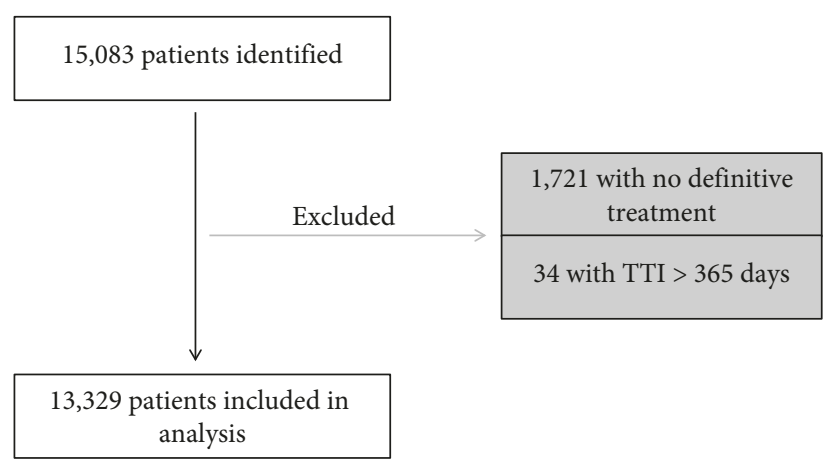

Figure 1: Inclusion and exclusion criteria (TTI, time to treatment initiation).

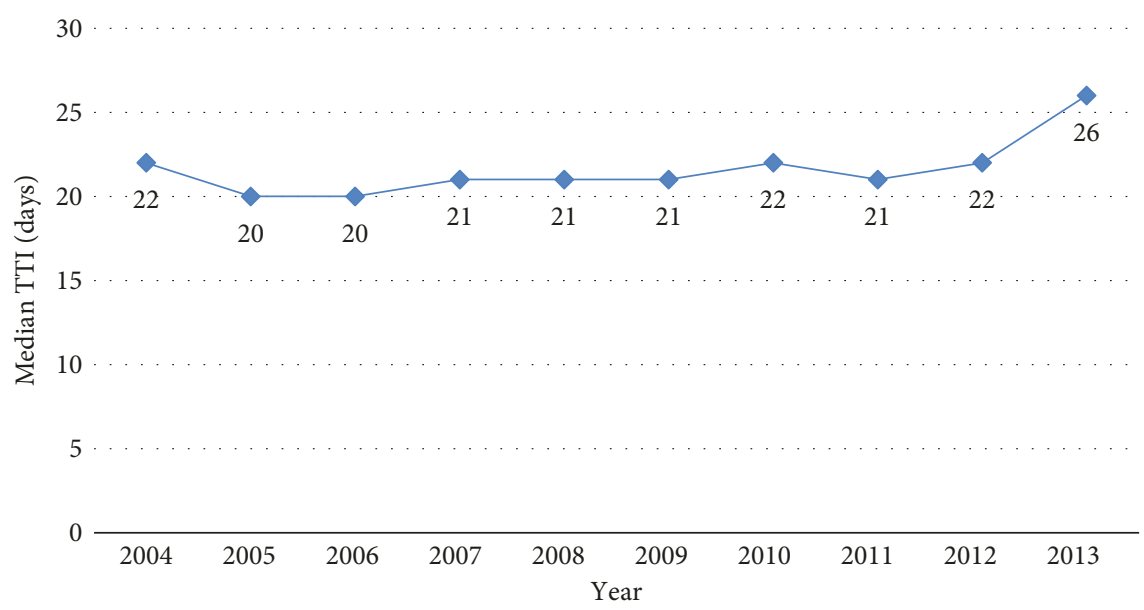

FIgure 2: Median time to treatment initiation by year from 2004 to 2013. $P$ value $=0.165$ (TTI, time to treatment initiation).

other anatomical sites. Intermediate grade tumors (grade 2 $($ IRR $=1.24 ; 95 \%$ CI, 1.12-1.38; $P<0.001))$ and higher grade tumors (grade $3 / 4 \quad(\mathrm{IRR}=1.13 ; 95 \% \mathrm{CI}, 1.03-1.23$; $P=0.006)$ ) correlated with increased TTI compared to grade 1 tumors. Patients with chondrosarcoma had significantly decreased TTI compared to all other diagnoses (IRR $=0.85 ; 95 \% \mathrm{CI}, 0.79-0.92 ; P<0.001)$. Other tumor factors that significantly decreased TTI include upper extremity tumors $(\mathrm{IRR}=0.92 ; 95 \% \mathrm{CI}, 0.85-0.99 ; P=0.023)$ and lower extremity tumors $(\mathrm{IRR}=0.91 ; 95 \% \mathrm{CI}, 0.86-0.96 ; P=0.001$ ) compared to all other primary tumor sites, and stage II/III $(\mathrm{IRR}=0.91 ; 95 \% \mathrm{CI}, 0.85-0.98 ; P=0.010)$ compared to all other stages. Larger tumor size $(\mathrm{IRR}=1.00 ; 95 \% \mathrm{CI}$, $0.95-1.05 ; P=0.923$ ), and an osteosarcoma diagnosis (IRR = 1.03 ; $95 \%$ CI, $0.97-1.09 ; P=0.316$ ) did not significantly influence TTI.

Healthcare system factors were shown to independently affect TTI. Academic centers were associated with increased TTI compared to all other centers (IRR $=1.14 ; 95 \% \mathrm{CI}$, $1.08-1.21 ; P<0.001)$ and especially when compared to only comprehensive cancer centers (IRR $=1.20$; 95\% CI, 1.11 $1.30 ; P<0.001)$. Compared to private insurance, uninsured $($ IRR $=1.36 ; 95 \%$ CI, 1.21-1.53; $P<0.001)$, Medicaid (IRR = 1.22 ; $95 \%$ CI, $1.12-1.33 ; P<0.001)$, or Medicare $($ IRR $=1.08$; 95\% CI, 1.01-1.16; $P=0.017)$ patients, all experienced significant TTI delays. A transition in care was the most significant predictor of increased TTI, when compared to patients without a transition in care (who received treatment at the same facility the diagnosis was established) (IRR = 1.89; 95\% CI, 1.80-1.99; $P<0.001)$. Surgery as the initial definitive treatment was the only factor associated with significantly decreased TTI when compared to all other treatment types $($ IRR $=0.88 ; 95 \% \mathrm{CI}, 0.84-0.94 ; P<0.001$ ). Living $>20$ miles from the facility did not significantly affect TTI compared to living $\leq 20$ miles of the facility ( IRR $=0.98$; 95\% CI, 0.93-1.03; $P=0.520$ ).

\section{Discussion}

TTI is gaining increased attention as a quality metric used by healthcare institutions for various cancer types, including lung, breast and head and neck cancer $[8,9,15]$. TTI initiatives are driven by patient demand, marketing, and the supposition that survival may be positively affected with expeditious treatment. Given the lack of reported TTI data in PBS, this study was intended to define the current national norms for TTI in adult patients with PBS in the United States, as well as to identify factors that may significantly increase or decrease TTI. This paper was not intended to link survival conclusions with TTI. These data show that, from 2004 to 2013, the median and mean TTI for PBS in the United States were 22 days and 32 days, respectively. As 
TABLE 1: Comparison of patient, tumor, and healthcare factors on time to treatment initiation.

\begin{tabular}{|c|c|c|c|}
\hline & Number of patients (\%) & Median TTI, days (IQR) & $P$ value \\
\hline Total number of patients & 13329 & $22(4-43)$ & \\
\hline Year of diagnosis & & & 0.0002 \\
\hline 2004 & $1189(9)$ & $22(3,43)$ & \\
\hline 2005 & $1339(10)$ & $20(0,42)$ & \\
\hline 2006 & $1258(9)$ & $20(2,41)$ & \\
\hline 2007 & $1307(10)$ & $21(2,45)$ & \\
\hline 2008 & $1290(10)$ & $21(3.75,42)$ & \\
\hline 2009 & $1393(11)$ & $21(2,43)$ & \\
\hline 2010 & $1370(10)$ & $22(5,46)$ & \\
\hline 2011 & $1349(10)$ & $21(4,43)$ & \\
\hline 2012 & $1451(11)$ & $22(4,43)$ & \\
\hline 2013 & $1383(10)$ & $26(7,48)$ & \\
\hline Age (range) & & & 0.0004 \\
\hline $18-30$ years & $3220(24)$ & $19(8,35)$ & \\
\hline $31-50$ years & $3882(29)$ & $22(0,44)$ & \\
\hline $51-70$ years & $4248(32)$ & $23(1,47)$ & \\
\hline $71+$ years & $1979(15)$ & $24(3,48)$ & \\
\hline Gender & & & 0.0017 \\
\hline Male & $7439(56)$ & $22(6,43)$ & \\
\hline Female & $5890(44)$ & $21(0,43)$ & \\
\hline Race & & & 0.0002 \\
\hline White & $11277(85)$ & $21(3,42)$ & \\
\hline Black & $1228(9)$ & $25(6,49.75)$ & \\
\hline Other/unknown & $824(6)$ & $21(3,45)$ & \\
\hline Charlson/Deyo score & & & 0.5091 \\
\hline 0 & $11433(86)$ & $22(4,43)$ & \\
\hline 1 & $1493(11)$ & $21(1,45)$ & \\
\hline$\geq 2$ & $403(3)$ & $24(0,54)$ & \\
\hline Income & & & 0.4598 \\
\hline$<\$ 38,000$ & $2216(17)$ & $22(5,46)$ & \\
\hline$\$ 38,000-\$ 47,999$ & $3130(24)$ & $22(4,44)$ & \\
\hline$\$ 48,000-\$ 62,999$ & $3504(26)$ & $21(3,42)$ & \\
\hline$\$ 63,000+$ & $4236(32)$ & $22(2.25,42)$ & \\
\hline Unknown & $243(2)$ & $21(6,40)$ & \\
\hline Histology & & & 0.0001 \\
\hline Osteosarcoma & $3979(30)$ & $24(10,42)$ & \\
\hline Chondrosarcoma & $5608(42)$ & $19(0,43)$ & \\
\hline Ewing's sarcoma & $1313(10)$ & $19(9,32)$ & \\
\hline Chordoma & $1354(10)$ & $30(1,63)$ & \\
\hline Other & $1075(8)$ & $25(7,49)$ & \\
\hline Primary tumor site & & & 0.0001 \\
\hline Upper extremity & $1743(13)$ & $20(0,40)$ & \\
\hline Lower extremity & $4482(34)$ & $20(7,37)$ & \\
\hline Pelvis & $2543(19)$ & $30(13,56)$ & \\
\hline Other & $4561(34)$ & $20(0,46)$ & \\
\hline Tumor size & & & 0.0001 \\
\hline$\leq 8.0 \mathrm{~cm}$ & $6397(48)$ & $21(0,44)$ & \\
\hline$>8.0 \mathrm{~cm}$ & $6932(52)$ & $22(7,43)$ & \\
\hline Grade & & & 0.0001 \\
\hline 1, well differentiated & 2488 (19) & $11.5(0,40)$ & \\
\hline 2 , moderately differentiated & $2246(17)$ & $26(3,49)$ & \\
\hline 3 or 4 , poorly/undifferentiated & $4300(33)$ & $23(10,41)$ & \\
\hline Unknown & $4295(32)$ & $22(4,45)$ & \\
\hline Clinical staging & & & 0.0001 \\
\hline Stage I & $4419(33)$ & $24(0,49)$ & \\
\hline Stage II & $2518(19)$ & $25(12,42)$ & \\
\hline Stage III & $216(2)$ & $23.5(10.25,45)$ & \\
\hline Stage IV & $1288(10)$ & $21(10,38)$ & \\
\hline Unknown & $4888(37)$ & $19(0,42)$ & \\
\hline
\end{tabular}


TABLE 1: Continued.

\begin{tabular}{|c|c|c|c|}
\hline & Number of patients (\%) & Median TTI, days (IQR) & $P$ value \\
\hline Facility type & & & 0.0001 \\
\hline Community cancer program & $303(2)$ & $22(0,43)$ & \\
\hline Comprehensive cancer center & $2026(15)$ & $19(0,41)$ & \\
\hline Academic center & $5652(42)$ & $26(5,49)$ & \\
\hline Integrated network cancer program & $544(4)$ & $20(0,44)$ & \\
\hline Other/unknown & $4804(36)$ & $20(6,38)$ & \\
\hline Insurance & & & 0.0001 \\
\hline Uninsured & $674(5)$ & $24(5.75,48)$ & \\
\hline Private insurance & $7488(56)$ & $20(1,41)$ & \\
\hline Medicaid & $1372(10)$ & $23(8,46)$ & \\
\hline Medicare & $2983(22)$ & $24(3,47)$ & \\
\hline Other/unknown & $812(6)$ & $28(9,52)$ & \\
\hline Distance from facility & & & 0.0001 \\
\hline$<21$ miles & $6238(47)$ & $21(1,42)$ & \\
\hline 21-50 miles & $2846(21)$ & $22(5,42)$ & \\
\hline $51-100$ miles & $1808(14)$ & $21(5,43)$ & \\
\hline$>100$ miles & $2212(17)$ & $25(6,48.75)$ & \\
\hline Unknown & $225(2)$ & $21(6,39.5)$ & \\
\hline Initial treatment modality & & & 0.0001 \\
\hline Surgery & $8949(67)$ & $19(0,44)$ & \\
\hline Radiation & $765(6)$ & $37(18,68.5)$ & \\
\hline Systemic & $3500(26)$ & $24(14,38)$ & \\
\hline Other & $24(0.2)$ & $30.5(2.5,68.25)$ & \\
\hline Multimodal & $91(0.7)$ & $23(6,49)$ & \\
\hline Transition in care & & & 0.0001 \\
\hline Yes & $5309(40)$ & $\begin{array}{c}34(17,58) \\
\text { Mean: } 44.9(43.7,46.1)\end{array}$ & \\
\hline No & $8020(60)$ & $\begin{array}{c}14(0,33) \\
\text { Mean: } 23.4(22.7,24.1)\end{array}$ & \\
\hline
\end{tabular}

Community Cancer Program volumes defined as 100-500 cancer cases annually. Integrated network cancer programs usually have a "unified cancer committee" and consist of "multiple facilities providing comprehensive services" [13]. Academic institutions are defined with the same quantitative volume definition as a comprehensive cancer center ( $>500$ cancer cases annually) but also have a noted resident/medical education tract supported.

a comparison, TTI for head and neck cancer in the United States was 26 days between 1998 and 2011 [9], while TTI for colon [16] and lung [17] cancer in France between 2009 and 2010 was 22 and 34 days, respectively. In the current study, Figure 3 delineates those factors that were attributed to increased or decreased TTI in PBS. As has been previously noted with soft tissue sarcoma, the single largest factor associated with increased TTI is a transition in care [12].

A transition in care significantly increased median TTI by nearly three weeks (34 days vs 14 days). This longer time period may be explained by the process of scheduling an appointment, obtaining insurance approval, and transferring records, including imaging and pathology. In addition, Mankin et al. notably reported that changes in management were two to twelve times greater when a part of the diagnostic or treatment process was performed at a referring facility compared to entirely at a single treatment facility, which also could lead to longer treatment times $[18,19]$. Further analysis of this dataset revealed an increasing proportion of PBS patients undergoing a transition of care in 2013 compared to $2004(437 / 1189=36.7 \%$ in 2004 compared to $609 / 1383=44.0 \%$ in $2013 ; P<0.001)$. This may signify a shift toward centralization of sarcoma cases to tertiary referral centers, which has been advocated [20-22].
While age, gender, comorbidities, income, and distance to the treating facility did not influence TTI, we found minority race and uninsured or government (Medicaid or Medicare) insurance status were all associated with increased TTI. Minority race and lower socioeconomic status are factors known to be associated with poorer access to care and worse survival in many types of cancer $[23,24]$. Uninsured patients were less likely to receive treatment compared to their insured counterparts in melanoma [25] and hepatocellular carcinoma [26]. While insurance status has previously been shown to not be associated with the incidence of incompletely excised soft tissue sarcoma and appropriate referral patterns [27], recent studies have revealed black populations did not receive the same frequency of surgical resection or radiation therapy [28] and were more likely to receive amputations, when compared to white populations with soft tissue sarcoma [29]. Although the specific reason for these associations is unknown, they are likely multifactorial in nature.

Treatment at academic centers was associated with increased TTI by nearly a week compared to community cancer programs and comprehensive cancer centers. We hypothesize that this is not a reflection of trainee involvement, as referral practices and major treatment 
TABle 2: Multivariate analysis.

\begin{tabular}{|c|c|c|}
\hline & Incidence rate ratio on TTI $(95 \% \mathrm{CI})$ & $P$ value \\
\hline Age (>30 years) & $1.07(1.00,1.14)$ & 0.051 \\
\hline Gender (female) & $0.99(0.94,1.04)$ & 0.612 \\
\hline Minority race & $1.12(1.04,1.19)$ & 0.002 \\
\hline Charlson/Deyo score $\geq 1$ & $0.98(0.92,1.06)$ & 0.677 \\
\hline \multicolumn{3}{|l|}{ Histology } \\
\hline Osteosarcoma & $1.03(0.97,1.09)$ & 0.316 \\
\hline Chondrosarcoma & $0.85(0.79,0.92)$ & $<0.001$ \\
\hline \multicolumn{3}{|l|}{ Primary tumor site } \\
\hline Upper extremity & $0.92(0.85,0.99)$ & 0.023 \\
\hline Lower extremity & $0.91(0.86,0.96)$ & 0.001 \\
\hline Pelvis & $1.26(1.19,1.35)$ & $<0.001$ \\
\hline \multicolumn{3}{|l|}{ Tumor size } \\
\hline$>8.0 \mathrm{~cm}$ & $1.00(0.95,1.05)$ & 0.923 \\
\hline \multicolumn{3}{|l|}{ Grade } \\
\hline Overall grade & $0.99(0.97,1.01)$ & 0.265 \\
\hline Grade 2 vs grade 1 & $1.24(1.12,1.38)$ & $<0.001$ \\
\hline Grade 3 or 4 vs grade 1 & $1.13(1.03,1.23)$ & 0.006 \\
\hline \multicolumn{3}{|l|}{ Clinical staging } \\
\hline Overall stage & $0.98(0.98,0.99)$ & $<0.001$ \\
\hline Stage II or III & $0.91(0.85,0.98)$ & 0.010 \\
\hline \multicolumn{3}{|l|}{ Facility type } \\
\hline Academic center & $1.14(1.08,1.21)$ & $<0.001$ \\
\hline Academic center vs comprehensive cancer center & $1.20(1.11,1.30)$ & $<0.001$ \\
\hline \multicolumn{3}{|l|}{ Insurance } \\
\hline Private insurance & $0.87(0.83,0.92)$ & $<0.001$ \\
\hline Uninsured vs private insurance & $1.36(1.21,1.53)$ & $<0.001$ \\
\hline Medicaid vs private insurance & $1.22(1.12,1.33)$ & $<0.001$ \\
\hline Medicare vs private insurance & $1.08(1.01,1.16)$ & 0.017 \\
\hline Distance to facility $\geq 21$ miles & $0.98(0.93,1.03)$ & 0.520 \\
\hline \multicolumn{3}{|l|}{ Initial treatment modality } \\
\hline Surgery & $0.88(0.84,0.94)$ & $<0.001$ \\
\hline Transition in care & $1.89(1.80,1.99)$ & $<0.001$ \\
\hline
\end{tabular}

decisions are independent of the educational program. Increased TTI at academic centers is likely related to the "oneway street" pattern of referral from a community setting to a tertiary-care academic center-common in rare diseases. As indicated by this dataset, academic centers have seen an increasing proportion of PBS patients over the past decade $(495 / 1189=41.6 \%$ in 2004 compared to $625 / 1383=45.2 \%$ in 2013; $P=0.069)$, which may be due to the increased prevalence of multidisciplinary sarcoma teams in academic centers and associated education efforts to centralize referral to these teams.

Primary tumors of the pelvis were also associated with increased TTI. Tumors in the pelvis and sacrum can be an extensive surgical undertaking, often requiring long surgical time, multiple surgical teams, and complex surgical techniques $[30,31]$. This additional coordination of care in pelvic sarcomas can undoubtedly lend itself to delays and the need for specialized resources-as evidenced by the fact that nearly half $(46.7 \%, 1189 / 2543)$ of pelvic sarcomas were seen at academic institutions in this population. In contrast, extremity-based tumors were associated with decreased TTI, likely attributed to a more straightforward approach toward treatment.
Higher-grade tumors (grades 2-4) had an increased TTI compared to low-grade tumors (grade 1). One hypothesis is that more locally aggressive tumors may require more complex resection and extensive surgical planning, particularly if surrounding neurovascular structures are involved. Alternatively, low-grade tumors can often be biopsied and resected in the same setting (e.g., long bone low-grade chondrosarcoma [32]), lending to decreased TTI (often $\mathrm{TTI}=0$ ). With cartilage lesions, radiographic diagnosis may drive clinical suspicion to plan wide resection for highergrade chondrosarcomas, with confirmatory frozen section at the time of surgery. This may offer an explanation as to why the diagnosis of chondrosarcoma and surgery as the index definitive treatment (includes excisional biopsies, TTI = 0 days) was associated with decreased TTI.

Lastly, it is interesting to note that though osteosarcoma and Ewing's sarcoma comprised $40 \%$ of this adult patient population, only $26 \%$ of patients had initial treatment with chemotherapy. Though the treatment specific data available through the NCDB did not offer any additional insight into this unique discrepancy, it is very thought provoking. One hypothesis for this finding may be as a result of the adult population ( $\geq 18$ years old) in this study, with the theory that 


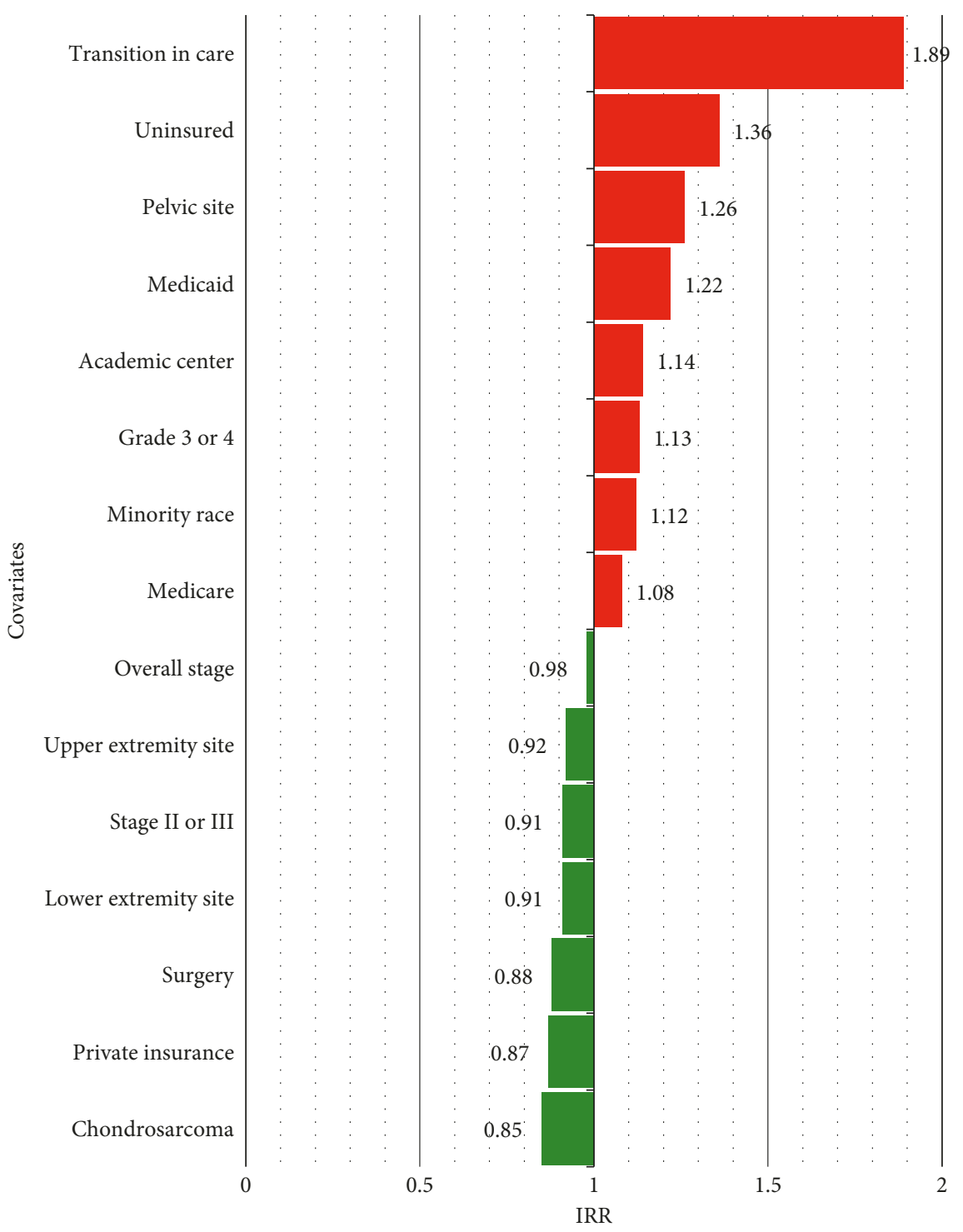

Figure 3: Comparison of relative association between covariates and time to treatment initiation. Only covariates with statistically significant higher (red) or lower (green) IRR are shown in this figure.

older osteosarcoma patients may be unable to tolerate chemotherapy to the same degree as younger patients, causing a larger proportion of patients to receive surgery as their initial treatment. Another factor that may contribute is that those with Paget's disease osteosarcoma are sometimes treated with up front resection prior to systemic therapy, given the aggressive nature of this variant.

This study has several limitations. First, data collection errors are inherent when using national registries, which can be attributed to incomplete and incorrect data found in medical charts. In addition, this NCDB search did not include patients $<18$ years old. This presents a selection bias away from certain subtypes of primary PBS, such as osteosarcoma and Ewing's sarcoma, as approximately 53\% of osteosarcoma cases have been reported to occur in patients $\leq 24$ years old [33]. Moreover, though the NCDB captures $70 \%$ of cancer cases, selection bias is possible in this database secondary to differences in institutions that report to the $\mathrm{NCDB}$. In the previous literature, incidence for PBS has been reported to be 0.9 per 100,000 people/year [34]. Assuming this incidence with knowledge of the USA population from 2004 to 2013, the total estimated number of PBS between 2004 and 2013 was 27,450 [35]. This would suggest approximately $55 \%$ of all new PBS were reported to the NCDB in this time frame, making it less common to report than other more common malignancies, and harder to appreciate definitive conclusions from the current data. Despite these limitations, this study provides a large patient cohort to assess this novel topic in PBS care.

\section{Conclusions}

PBS has a median TTI of 22 days and a mean TTI of 32 days. In addition, socioeconomic, tumor, treatment, and healthcare 
system factors were found to significantly affect TTI. Potential causes of prolongation of TTI are multifactorial in nature. Using the information provided in this study, physicians can potentially identify their own institutional processes linked to treatment delays, with the goal to streamline treatment, improve patient care, and reduce patient anxiety. Future studies should assess the prognostic risk that delays in TTI pose on patient survival in PBS.

\section{Data Availability}

The data used to support the findings of this study are included within the article.

\section{Conflicts of Interest}

The authors declare no relevant or conflicting interests related to this work.

\section{References}

[1] Bone Cancer: Statistics, CancerNet, May 2018, https://www. cancer.net/cancer-types/bone-cancer/statistics.

[2] M. P. Link, A. M. Goorin, A. W. Miser et al., "The effect of adjuvant chemotherapy on relapse-free survival in patients with osteosarcoma of the extremity," New England Journal of Medicine, vol. 314, no. 25, pp. 1600-1606, 1986.

[3] F. Eilber, A. Giuliano, J. Eckardt, K. Patterson, S. Moseley, and J. Goodnight, "Adjuvant chemotherapy for osteosarcoma: a randomized prospective trial," Journal of Clinical Oncology, vol. 5, no. 1, pp. 21-26, 1987.

[4] N. Jaffe, "Osteosarcoma: review of the past, impact on the future. The American experience," Cancer Treatment and Research, vol. 152, pp. 239-262, 2009.

[5] R. J. Grimer, "Size matters for sarcomas!," The Annals of the Royal College of Surgeons of England, vol. 88, no. 6, pp. 519-524, 2006.

[6] G. Smith, G. Johnson, R. Grimer, and S. Wilson, "Trends in presentation of bone and soft tissue sarcomas over 25 years: little evidence of earlier diagnosis," The Annals of the Royal College of Surgeons of England, vol. 93, no. 7, pp. 542-547, 2011.

[7] C. Schnurr, M. Pippan, H. Stuetzer, K. S. Delank, Michael JWP, and P. Eysel, "Treatment delay of bone tumours, compilation of a sociodemographic risk profile: a retrospective study," BMC Cancer, vol. 8, no. 1, p. 22, 2008.

[8] R. J. Bleicher, K. Ruth, E. R. Sigurdson et al., "Time to surgery and breast cancer survival in the United States," JAMA Oncology, vol. 2, no. 3, pp. 330-339, 2016.

[9] C. T. Murphy, T. J. Galloway, E. A. Handorf et al., "Increasing time to treatment initiation for head and neck cancer: an analysis of the National Cancer Database," Cancer, vol. 121, no. 8, pp. 1204-1213, 2015.

[10] C. T. Murphy, T. J. Galloway, E. A. Handorf et al., "Survival impact of increasing time to treatment initiation for patients with head and neck cancer in the United States," Journal of Clinical Oncology, vol. 34, no. 2, pp. 169-178, 2016.

[11] S. Mohanty and K. Y. Bilimoria, "Comparing national cancer registries: the National Cancer Data Base (NCDB) and the Surveillance, Epidemiology, and End Results (SEER) program," Journal of Surgical Oncology, vol. 109, no. 7, pp. 629-630, 2014.
[12] G. Curtis, J. Lawrenz, J. George et al., "Adult soft tissue sarcoma and time to treatment initiation: an analysis of the National Cancer Database," Journal of Surgical Oncology, vol. 117 , no. 8 , pp. 1776-1785, 2018, In press.

[13] National Cancer Data Base-Data Dictionary PUF 2014 n.d., 2017, http://ncdbpuf.facs.org/node/259?q=print-pdf-all.

[14] K. Y. Bilimoria, A. K. Stewart, D. P. Winchester, and C. Y. Ko, "The National Cancer Data Base: a powerful initiative to improve cancer care in the United States," Annals of Surgical Oncology, vol. 15, no. 3, pp. 683-690, 2008.

[15] P. Samson, A. Patel, T. Garrett et al., "Effects of delayed surgical resection on short-term and long-term outcomes in clinical stage I non-small cell lung cancer," Annals of Thoracic Surgery, vol. 99, no. 6, pp. 1906-1913, 2015.

[16] Y. C. Kudjawu, D. Eilstein, E. Decool, F. De Maria, N. Beltzer, and G. Chatellier, "Time to first treatment after colonoscopy in patients suffering from colon or rectum cancer in France," Cancer Epidemiology, vol. 39, no. 6, pp. 877-884, 2015.

[17] Y. C. Kudjawu, G. Chatellier, E. Decool et al., "Timing in initiating lung cancer treatment after bronchoscopy in France: study from medico-administrative database," Lung Cancer, vol. 95, pp. 44-50, 2016.

[18] H. J. Mankin, T. A. Lange, and S. S. Spanier, "The hazards of biopsy in patients with malignant primary bone and softtissue tumors," Journal of Bone \& Joint Surgery, vol. 64, no. 8, pp. 1121-1127, 1982.

[19] H. J. Mankin, C. J. Mankin, and M. A. Simon, "The hazards of the biopsy, revisited.: for the members of the Musculoskeletal Tumor Society," Journal of Bone \& Joint Surgery, vol. 78, no. 5, pp. 656-663, 1996.

[20] J. C. Gutierrez, E. A. Perez, F. L. Moffat, A. S. Livingstone, D. Franceschi, and L. G. Koniaris, "Should soft tissue sarcomas be treated at high-volume centers? An analysis of 4205 patients," Annals of Surgery, vol. 245, no. 6, pp. 952-958, 2007.

[21] H. J. Hoekstra, R. L. M. Haas, C. Verhoef et al., "Adherence to guidelines for adult (Non-GIST) soft tissue sarcoma in The Netherlands: a plea for dedicated sarcoma centers," Annals of Surgical Oncology, vol. 24, no. 11, pp. 3279-3288, 2017.

[22] P. Gustafson, K. E. Dreinhöfer, and A. Rydholm, "Soft tissue sarcoma should be treated at a tumor center: a comparison of quality of surgery in 375 patients," Acta Orthopaedica Scandinavica, vol. 65, no. 1, pp. 47-50, 1994.

[23] J. D. Albano, E. Ward, A. Jemal et al., "Cancer mortality in the United States by education level and race," Journal of the National Cancer Institute, vol. 99, no. 18, pp. 1384-1394, 2007.

[24] E. Ward, A. Jemal, V. Cokkinides et al., "Cancer disparities by race/ethnicity and socioeconomic status," CA: A Cancer Journal for Clinicians, vol. 54, no. 2, pp. 78-93, 2004.

[25] A. Amini, C. G. Rusthoven, T. V. Waxweiler et al., "Association of health insurance with outcomes in adults ages 18 to 64 years with melanoma in the United States," Journal of the American Academy of Dermatology, vol. 74, no. 2, pp. 309316, 2016.

[26] J. Wang, J. Ha, A. Lopez, T. Bhuket, B. Liu, and R. J. Wong, "Medicaid and uninsured hepatocellular carcinoma patients have more advanced tumor stage and are less likely to receive treatment," Journal of Clinical Gastroenterology, vol. 52, no. 5, pp. 437-443, 2017.

[27] V. K. Alamanda, G. O. Delisca, K. R. Archer, Y. Song, H. S. Schwartz, and G. E. Holt, "Incomplete excisions of extremity soft tissue sarcomas are unaffected by insurance status or distance from a sarcoma center," Journal of Surgical Oncology, vol. 108, no. 7, pp. 477-480, 2013. 
[28] V. K. Alamanda, Y. Song, H. S. Schwartz, and G. E. Holt, "Racial disparities in extremity soft-tissue sarcoma outcomes: a nationwide analysis," American Journal of Clinical Oncology, vol. 38, no. 6, pp. 595-599, 2015.

[29] A. L. Lazarides, J. D. Visgauss, D. P. Nussbaum et al., "Race is an independent predictor of survival in patients with soft tissue sarcoma of the extremities," BMC Cancer, vol. 18, no. 1 , p. $488,2018$.

[30] M. K. Laitinen, M. C. Parry, J. I. Albergo, R. J. Grimer, and L. M. Jeys, "Is computer navigation when used in the surgery of iliosacral pelvic bone tumours safer for the patient?," Bone \& Joint Journal, vol. 99-B, no. 2, pp. 261-266, 2017.

[31] A. F. Mavrogenis, K. Soultanis, P. Patapis et al., "Pelvic resections," Orthopedics, vol. 35, pp. e232-e243, 2012.

[32] E. F. Dierselhuis, J. G. Gerbers, J. J. W. Ploegmakers, M. Stevens, A. J. H. Suurmeijer, and P. C. Jutte, "Local treatment with adjuvant therapy for central atypical cartilaginous tumors in the long bones: analysis of outcome and complications in one hundred and eight patients with a minimum follow-up of two years," Journal of Bone and Joint Surgery, vol. 98, no. 4, pp. 303-313, 2016.

[33] L. Mirabello, R. J. Troisi, and S. A. Savage, "Osteosarcoma incidence and survival rates from 1973 to 2004," Cancer, vol. 115, no. 7, pp. 1531-1543, 2009.

[34] A. Franchi, "Epidemiology and classification of bone tumors," Clinical Cases in Mineral and Bone Metabolism, vol. 9, pp. 92-95, 2012.

[35] multpl. US Population by Year n.d. , May 2018, http://www. multpl.com/united-states-population/table. 


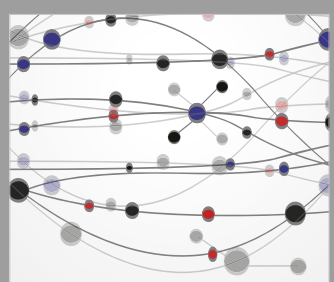

The Scientific World Journal
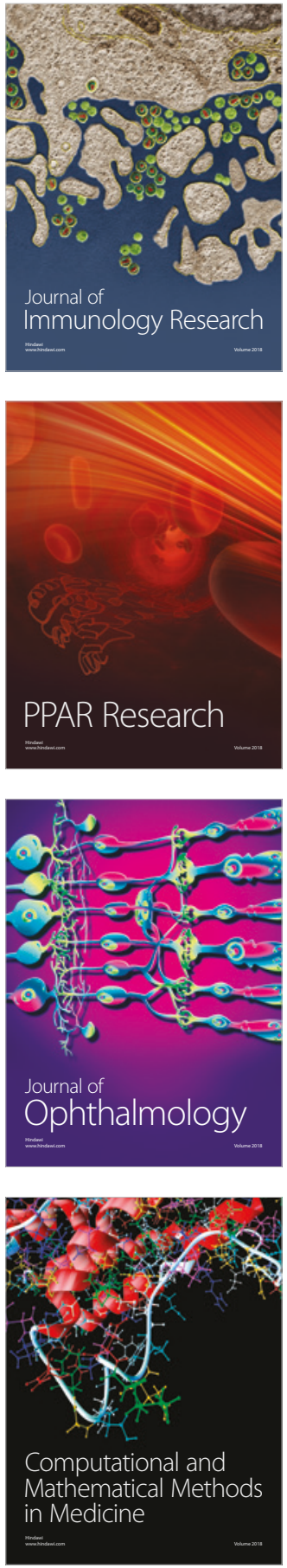

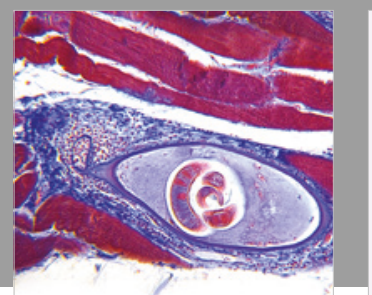

Gastroenterology Research and Practice

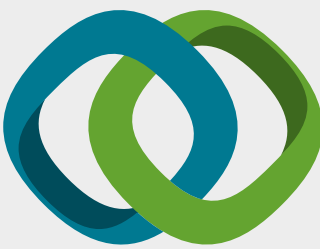

\section{Hindawi}

Submit your manuscripts at

www.hindawi.com
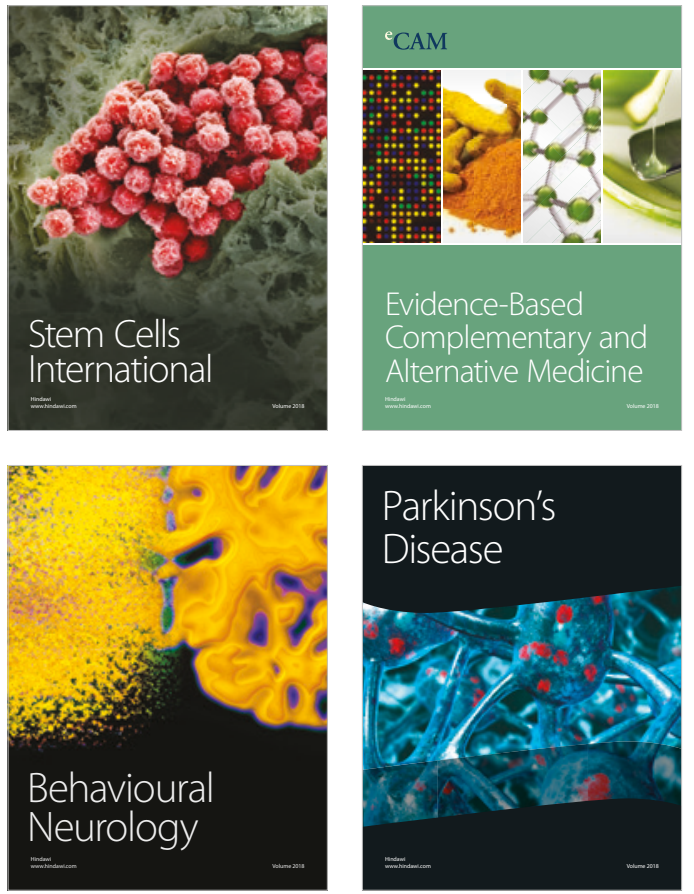

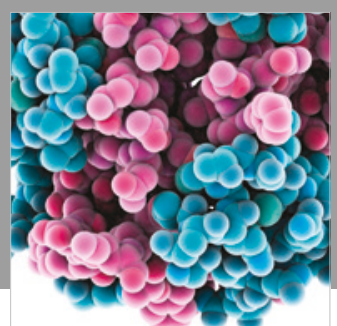

ournal of

Diabetes Research

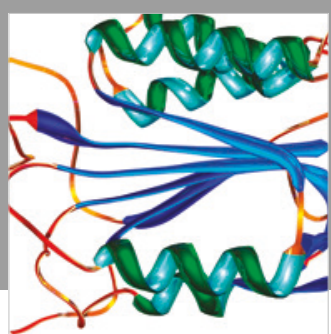

Disease Markers
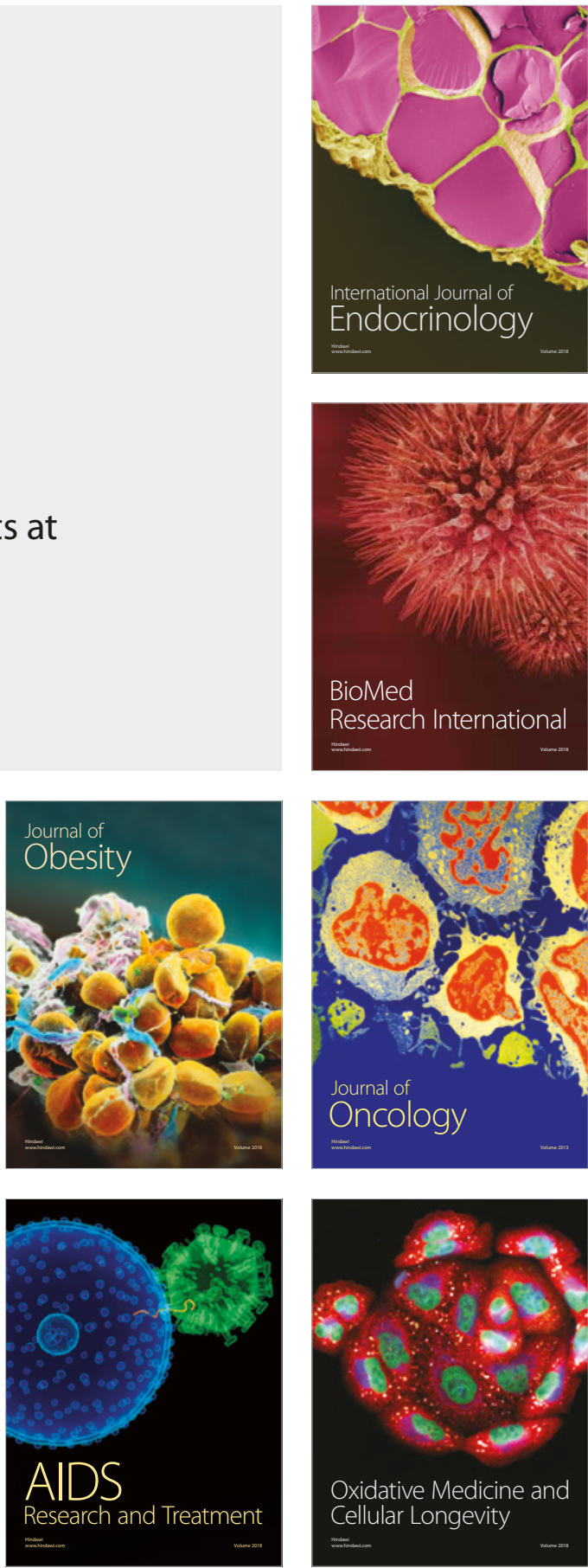